\title{
CORRECTION
}

Check for updates

Cite this: Phys. Chem. Chem. Phys., 2020, 22, 1785

DOI: $10.1039 / c 9 c p 90305 d$

rsc.li/pccp

\section{Correction: Plasma-induced grafting of polyacrylamide on graphene oxide nanosheets for simultaneous removal of radionuclides}

\author{
Wencheng Song, ${ }^{\mathrm{ab}}$ Xiangxue Wang, ${ }^{\mathrm{b}}$ Qi Wang, ${ }^{\mathrm{b}}$ Dadong Shao ${ }^{\mathrm{b}}$ and \\ Xiangke Wang*acd
}

Correction for 'Plasma-induced grafting of polyacrylamide on graphene oxide nanosheets for simultaneous removal of radionuclides' by Wencheng Song et al., Phys. Chem. Chem. Phys., 2015, 17, $398-406$

The authors regret that the Raman in Fig. 2c and XRD in Fig. 2e of the above mentioned paper were incorrect because of confusion with other samples. Besides, the labels of the XPS N 1s spectra of PAM/GO and PAM/GO-Eu were mislabeled in Fig. 7b, and the XPS $\mathrm{N}$ 1s spectra of PAM/GO was reanalyzed (Table S6). Thus, the Raman of GO and GO/PAM, XRD of PAM/GO and high-resolution XPS $\mathrm{N}$ 1s spectra of PAM/GO and PAM/GO-Eu were corrected below. The correction has no effect to the discussion and main conclusion.

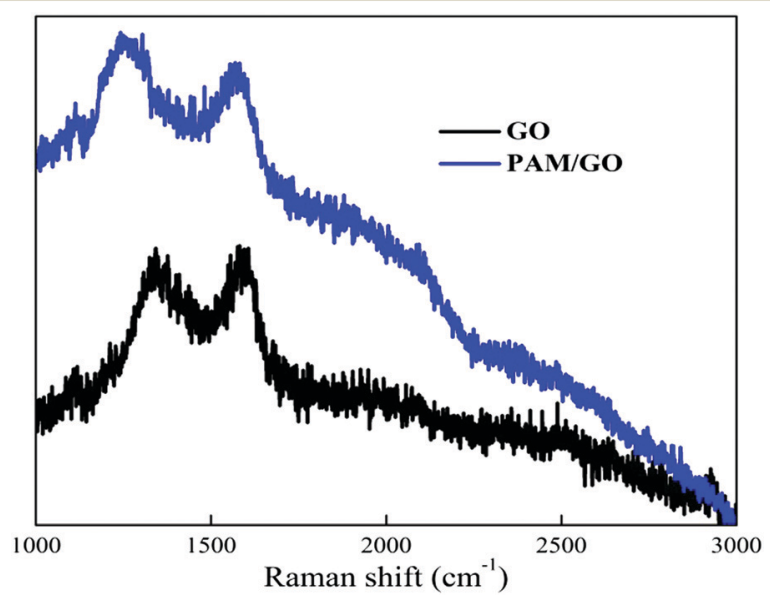

Fig. 2c Raman spectra of GO

\footnotetext{
${ }^{a}$ School of Environment and Chemical Engineering, North China Electric Power University, Beijing 102206, P. R. China. E-mail: xkwang@ipp.ac.cn; Fax: +86-551-65591310; Tel: $+86-551-65592788$

${ }^{b}$ Institute of Plasma Physics, Chinese Academy of Sciences, P.O. Box 1126, 230031, Hefei, China

${ }^{c}$ Faculty of Engineering, King Abdulaziz University, Jeddah 21589, Saudi Arabia

${ }^{d}$ Collaborative Innovation Center of Radiation Medicine of Jiangsu Higher Education Institutions, P. R. China
} 


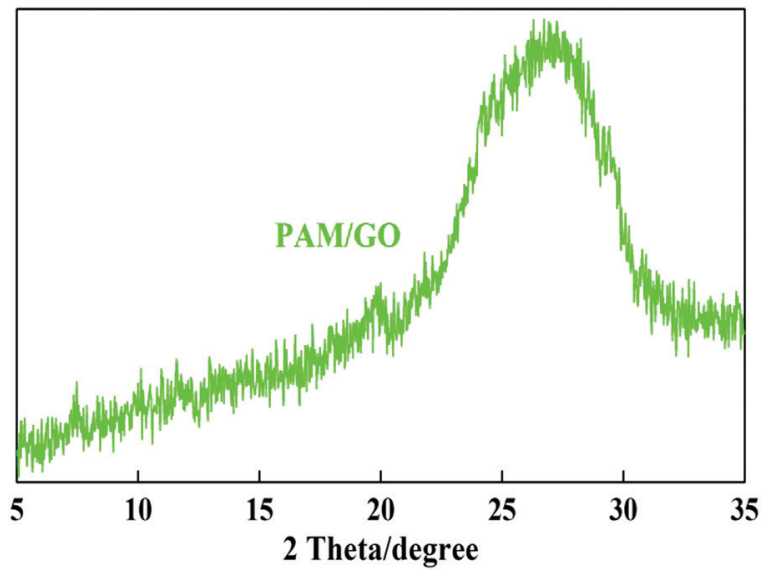

Fig. $2 e \quad X R D$ of PAM/GO.

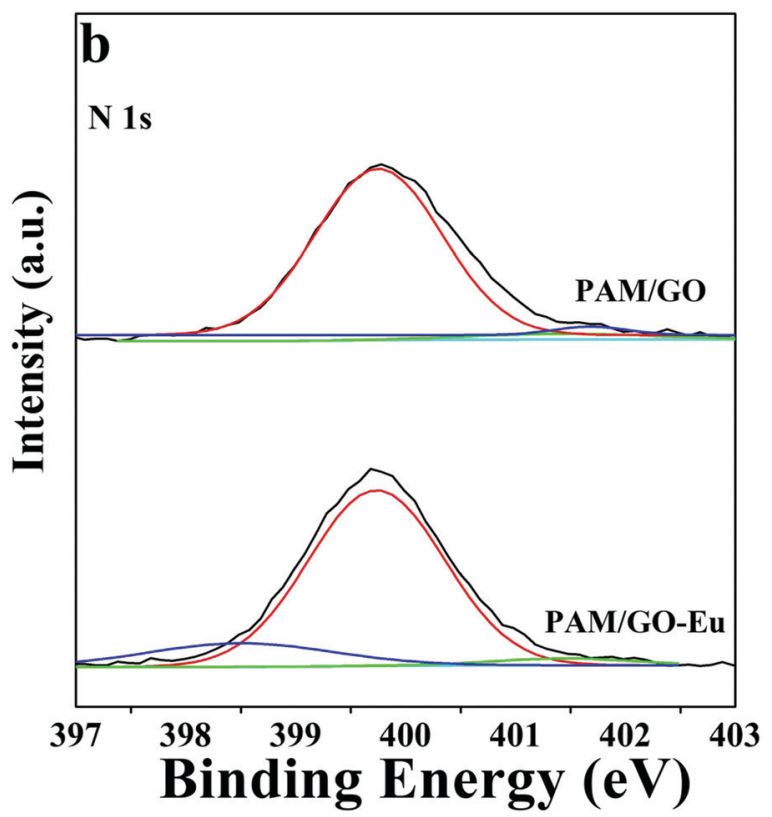

Fig. 7b High-resolution XPS N 1s spectra of PAM/GO and PAM/GO-Eu.

The revised curve fitting results of the XPS N 1s spectra can be found in Table S6 of the updated electronic supplementary information file alongside the original article and are also shown below:

Table S6 Curve fitting results of XPS N 1s spectra

\begin{tabular}{|c|c|c|c|c|c|}
\hline \multicolumn{2}{|l|}{ Type } & $\frac{\text { Peak }}{1}$ & $\frac{\mathrm{BE}(\mathrm{eV})}{399.92}$ & $\frac{F W H M(e V)}{1.88}$ & $\frac{\%}{93.54}$ \\
\hline & & 2 & 401.52 & 1.56 & 6.46 \\
\hline & & 3 & 401.44 & 1.39 & 4.27 \\
\hline
\end{tabular}

The Royal Society of Chemistry apologises for these errors and any consequent inconvenience to authors and readers. 